\section{Overcalling secondary findings}

To the Editor: Although I applaud the article by Jang et al. ${ }^{1}$ and their efforts to evaluate the incidence of secondary findings among Koreans, some of the conclusions should be reconsidered. First, they claim a 7\% rate of secondary findings, but this should have been accompanied by a confidence interval assessment because theirs is a (presumably) random sample population estimate. The $95 \%$ confidence interval (using the modified Wald method) of a proportion of 13 of 196 individuals with secondary findings gives a range of $3.8-11 \%$. The lower end of this range is not far from the estimated 3-4\% recommended by another publication, ${ }^{2}$ but the upper part of this range is highly implausible-it is not reasonable to suggest that 1 out of 10 people has a pathogenic mutation in these 56 genes. A much larger study of 4,300 individuals from the ESP sequencing project concluded that the overall yield for European Americans was approximately $2 \%$ (95\% confidence interval: $1.7-2.5 \%)$. Using a Fisher's exact test comparison, the yield of secondary findings in these two groups is highly significant $(P=0.0004)$, and I am aware of no epidemiologic data that would suggest that Koreans are overall 3.5 times more likely to have one of these diseases than are Americans of European origin.

An alternative hypothesis is that these variants were deemed pathogenic by criteria that were not sufficiently stringent and that Jang et al. ${ }^{1}$ misinterpreted some of the guidelines from the American College of Medical Genetics and Genomics (ACMG) incidental findings recommendations. ${ }^{2}$ For example, the ACMG secondary findings guidelines state that only known pathogenic mutations in RYR1 should be reported as incidental findings. Yet Jang et al. ${ }^{1}$ reported an apparently novel truncating mutation, c.5701C > T (p.Gln1901X), as likely pathogenic and included it in their yield. The malignant hyperthermia phenotype is nearly entirely associated with missense mutations, and no truncating mutations in RYR1 have been determined to be pathogenic by the European Malignant Hyperthermia Group (https://emhg.org/genetics/mutations-in-ryr1/). This mutation should not have been included in the authors' report because it is much more likely to be a loss-of-function mutation that would cause myopathy if found in trans to another RYR1 lossof-function allele and thus represents carrier status for a recessive disorder not included in the ACMG recommendations.

The authors also report a mutation that they describe as c.799C > T (p.Gln267X) and cite a paper in support of the pathogenicity of that variant. That variant is listed by the Human Gene
Mutation Database as "DM?" (which is a very strong predictor that it is not pathogenic), and the cited article (by Kim et al. ${ }^{3}$ ) describes that variant as being pathogenic in trans to another MUTYH variant. MUTYH-associated cancer phenotypes can exhibit both dominant and recessive modes of inheritance, and the finding of a single variant of the latter type is not reportable as an incidental (secondary) finding according to the ACMG recommendations, ${ }^{2}$ regardless of the pathogenicity.

It is not possible to enumerate all the errors of interpretation in the article by Jang et al., ${ }^{1}$ but the most likely explanation for their inflated estimate of secondary findings is that they have an inappropriately low threshold for reporting secondary variants. Although it is laudable to study secondary findings, it is critical that the standards of interpretation be high because lax standards will lead to excess return of false-positive secondary findings variants, which could lead to incorrect diagnoses, unnecessary patient or research participant distress, unnecessary follow-up evaluations, or potentially harmful medical or surgical interventions.

\section{ACKNOWLEDGMENTS}

The author is supported by the Intramural Research Program of the National Human Genome Research Institute of the National Institutes of Health. The opinions and evaluation described do not necessarily represent the official views of this institution.

\section{DISCLOSURE}

The author is an uncompensated adviser to the Illumina Corp. as a part of his official duties.

\section{Leslie G. Biesecker, $M D^{1}$}

${ }^{1}$ Medical Genomics and Metabolic Genetics Branch, National Human Genome Research Institute, National Institutes of Health, Bethesda, Maryland, USA. Correspondence: Leslie G. Biesecker (lesb@mail.nih.gov)

\section{REFERENCES}

1. Jang MA, Lee SH, Kim N, Ki CS. Frequency and spectrum of actionable pathogenic secondary findings in 196 Korean exomes. Genet Med 2015;17:1007-1011.

2. Green RC, Berg JS, Grody WW, et al.; American College of Medical Genetics and Genomics. ACMG recommendations for reporting of incidental findings in clinical exome and genome sequencing. Genet Med 2013;15:565-574.

3. Kim DW, Kim IJ, Kang HC, et al. Germline mutations of the MYH gene in Korean patients with multiple colorectal adenomas. Int J Colorectal Dis 2007;22: 1173-1178.

Advance online publication 17 March 2016. doi:10.1038/gim.2016.19 